UCRL-JC-119202

PREPRINT

\title{
Impact of Three-dimensional Nonuniformity on the Germanium X-ray Laser Output
}

\author{
A. S. Wan, R. W. Mayle, A. L. Osterheld \\ Lawrence Livermore National Laboratory \\ Livermore, CA 94550, USA \\ Y. Kato \\ Institute of Laser Engineering \\ Osaka University, Suita, Osaka 565, Japan
}

This paper.was prepared for submittal to the Proceedings of the 12th International Conference on Laser

Interaction and Related Plasma Phenomena

Osaka, Japan, April 24-28, 1995

April 1995

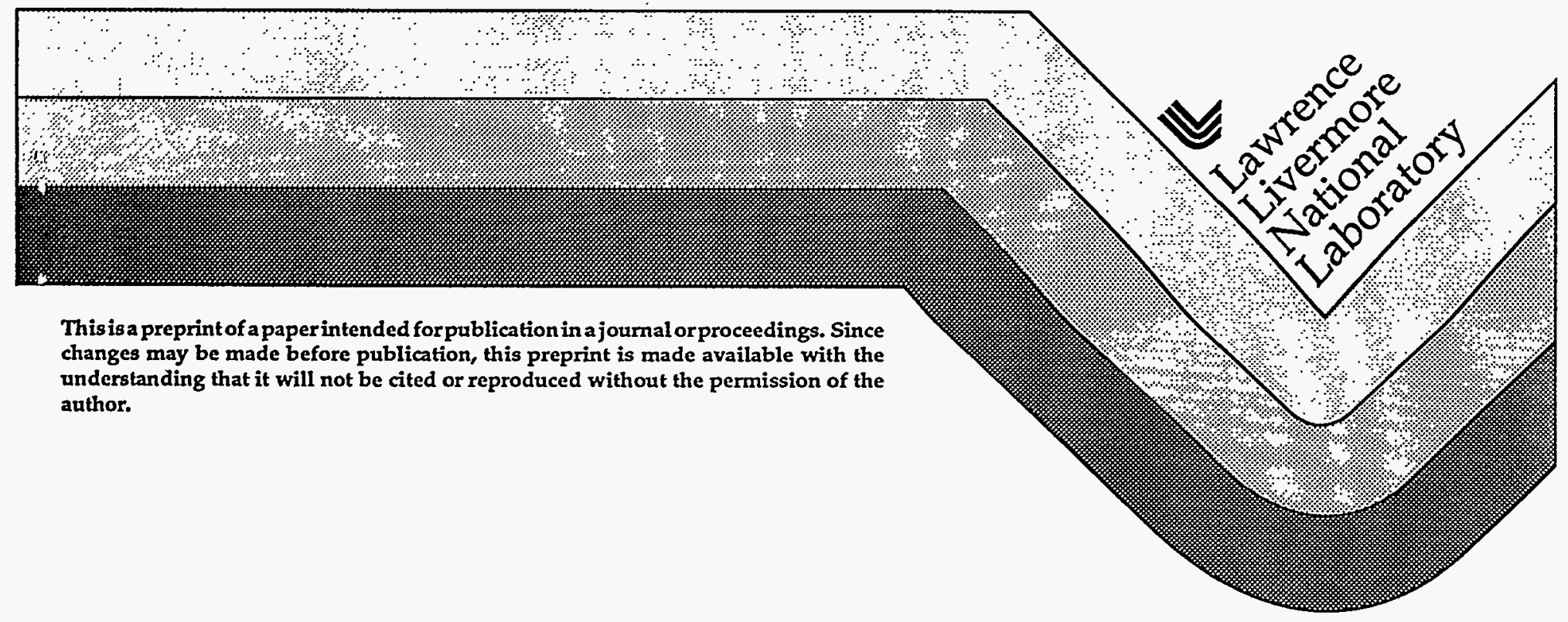




\section{DISCLAIMER}

This report was prepared as an account of work sponsored by an agency of the United States Government. Neither the United States Government nor any agency thereof, nor any of their employees, make any warranty, express or implied, or assumes any legal liability or responsibility for the accuracy, completeness, or usefulness of any information, apparatus, product, or process disclosed, or represents that its use would not infringe privately owned rights. Reference herein to any specific commercial product, process, or service by trade name, trademark, manufacturer, or otherwise does not necessarily constitute or imply its endorsement, recommendation, or favoring by the United States Government or any agency thereof. The views and opinions of authors expressed herein do not necessarily state or reflect those of the United States Government or any agency thereof. 


\section{DISCLAIMER}

Portions of this document may be illegible in electronic image products. Images are produced from the best available original document. 


\title{
Impact of Three-dimensional Nonuniformity on the Germanium X-ray Laser Output
}

\author{
A. S. Wan, R. W. Mayle, Y. Kato*, A. L. Osterheld \\ Lawrence Livermore National Laboratory, Livermore, CA 94550, U.S.A. \\ * Institute of Laser Engineering, Osaka University, Suita, Osaka 565, Japan
}

\begin{abstract}
We are studying the impact of axial nonuniformity of the driving optical laser on the output of $x$-ray lasers. In this paper we use typical laser focusing parameters from germanium $\mathrm{x}$-ray laser experiments on Gekko XII which has a bowtie-shaped line focus that results in large axial differences of the incident intensity on target. This axial nonuniformity of the driving laser intensity produced significant axial variations of spatial gain and density profiles. In this paper we contrast the $x$-ray laser output of axially homogeneous and in homogeneous germanium $x$-ray lasers using the numerical simulation code, XRASER, which includes the effect of laser photon transport, saturation, and refractive propagation in three spatial dimensions and two angular directions of photon phase space.
\end{abstract}

\section{INTRODUCTION}

In collisionally excited x-ray lasers (XRLs), high-temperature and high-density plasmas are produced by line-focused optical laser heating of foil or slab targets. The upper laser levels are preferentially populated by monopole coupled collisional excitation processes, but these levels are not radiatively coupled to the ground states. The fast depopulation of lower laser levels by resonance emission produces the population inversion. The population inversion depends sensitively on collisional and radiative processes and is a strong function of plasma conditions.

Plasma expands away from the target surface with large density gradients both parallel and perpendicular to the target surface. XRL photons propagate in this highly refractive gain medium, results in a large divergence output. Various methods to compensate for the effect of refraction in the direction perpendicular to the $x$-ray laser axis have been proposed and successfully demonstrated. $[1,2]$ However, an important consideration which has been neglected in the past is the impact of three-dimensional (3-D) nonuniformity on the output of XRLs.

We typically use a cylindrical lens and an axially symmetric focusing lens to generate a line-focused geometry for XRL experiments. However, the line focus generated may not a straight line along the XRL propagation axis (defined as the axial direction), but is curved which results in a bowtie-shaped focusing geometry as shown in Fig. 1. [3] Variation of the width of the line focus leads to large axial nonuniformity of the irradiance. Therefore, we should not neglect the axial gain and electron density $\left(n_{e}\right)$ variations when we model the XRL output performance. In this paper we study the impact of the 3-D nonuniformity on the output of Ge 


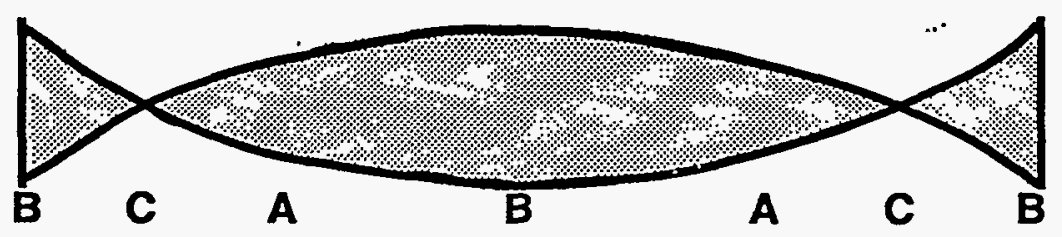

Figure 1. Bowtie-shaped line focus results in axially nonuniform gain and $n_{e}$ profiles due to irradiance variations. Letters mark the irradiance geometry listed in Table 1.

XRLs as the result of the axial irradiance variation with the simulation geometry matching the laser specification of the Gekko XII Ge XRL experiments.

\section{NUMERICAL MODEL AND SIMULATION GEOMETRY}

In modeling XRLs, we need to take into consideration the physics of laser-matter interaction, radiation hydrodynamics, radiation transfer, and non-LTE atomic kinetics. For collisionally dominated plasmas we can decouple the detailed level populations and line transfer physics from the rest of the problem. We use LASNEX [4] to carry out the laser-deposition and hydrodynamics simulations. From 2-D LASNEX calculations, we obtain 2-D profiles of plasma characteristics, such as $T_{e}$ and $n_{e}$, and variables such as mesh positions and velocities. We then postprocess LASNEX output using non-LTE atomic kinetic codes such as XRASER $[5,6]$ to calculate the level populations and line transfer.

XRASER separates the radiation into three components: continuum, non-lasing lines, and lasers. The continuum is treated with formal transfer. The (non-lasing) lines transfer enforces consistency between populations and line strengths through a complete linearization procedure. And the laser transport accounts for the effect of amplification and saturation using an iterative procedure in multiple dimensions. Figure 2 shows the 3-D simulation geometry in Cartesian coordinates which can be thought of as a collection of 2-D slices arranged along the lasing $z$-axis. The 3-D laser transport package [7] includes the effect of refractive propagation and is the only bridge of communication between the 2-D slices. XRASER solves the time dependent system determining the atomic populations self-consistently with the time dependent radiation field. We fully take into account the effect of gain saturation in determining the upper and lower levels of the lasing transition by solving for the combined system of matter and radiation. Before running the 3-D calculation, we need to re-map the information needed for XRASER from the distorted LASNEX Lagrangian mesh to a new rectangular mesh. The new mesh is chosen to be coarse, to save computer time, but yet sufficiently detailed such that there is no significant loss in the 2-D information which can affect the 3-D outcome.

A 3-D calculation which simulates an actual XRL configuration is both cpu and memory intensive. For the type of problems discussed in this paper, each 3-D simulation requires a minimum of $20 \mathrm{cpu}$ hours on a Cray super-computer. Most of the cpu is spent in the calculation of the atomic populations; we need to invert a large matrix as well as construct the matrix elements and we do this at each spatial point in our 3-D grid. The accuracy of the calculation is only as good as the atomic model. In addition to detailed $n=3$ levels in $\mathrm{Ne}$-like $\mathrm{Ge}$, where the population inversion occurs, we typically buffer these detailed levels with more detailed levels 


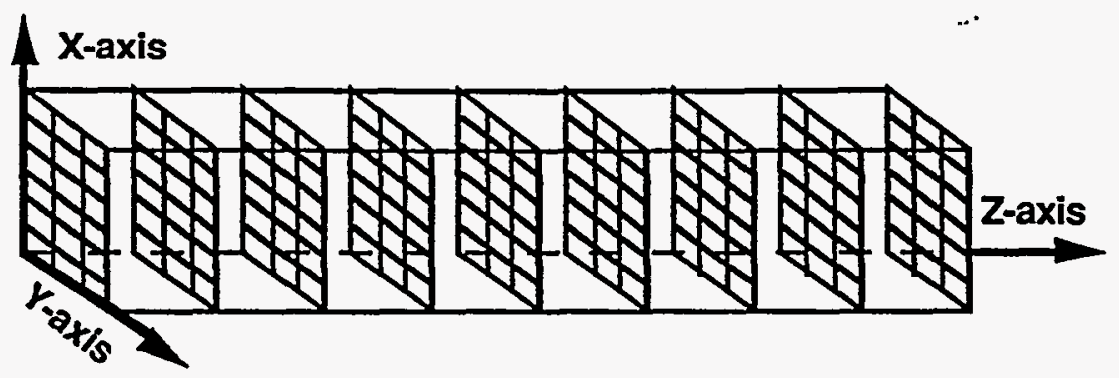

Figure 2. Geometry and coordinates used for the 3-D laser transport calculation.

in Na- and F-like isoelectronic sequences. However, to use a highly detailed model in a 3-D calculation would be prohibitively expensive and memory intensive. In our calculations we used a "simplified" Ge atomic model with 70 levels, with highly averaged levels buffering the detail $n=3$ Ne-like levels. 0 -D comparisons between the 200-level model and the 70-level model, at appropriate temperature and density regimes, indicate small changes in gain. However, in an amplifying medium any error in gain will be propagated exponentially. Therefore, the accuracy of the atomic model remains one of the major uncertainty in this study.

The number of "minimum" 2-D slices we must use in a 3-D laser simulation is sensitively dependent on the gain-length product of the XRL medium. The rule-ofthumb is to keep a gain-length product of $\sim 1$ between slices. However, in order to simulate a 3-cm-long Ge XRL and not to exceed the total memory available on the computer, we used a total of eleven slices, with $0.3 \mathrm{~cm}$ separation between slices. As shown in the next section, the calculated Ge XRL gain can be as large as $10 \mathrm{~cm}^{-}$ 1. The large gain-length product between slices mean that the amplification between slices can be as large as an order of magnitude. This creates further uncertainty to our simulation. Another large uncertainty of the 3-D result is from the angular bining of the rays in 3-D which is limited by cpu and memory. We define the angular bins to favor a long, thin geometry expected from a line-focused XRL. However, in such a highly refractive medium large angle rays are also very important. We are planning a series of simulations to study the effect of coarse spatial meshes (both axial and $x-y$ ) and angular bining on XRL output.

\section{IMPACT OF 3-D NONUNIFORMITY ON XRL OUTPUT}

Table 1 lists three different irradiance configurations of a driving laser illuminating a thick Ge slab. Each configuration is defined by the full width at half maximum (FWHM) of the gaussian spatial profile and the peak intensity of the laser pulse. These configurations, marked as A, B, and C in Fig. 1 of the Gekko line focus, correspond to planes transverse to the $\mathrm{XRL}$ propagation direction. Case $\mathrm{B}$ corresponds to the central and tail parts of the line focus with a wide focus and therefore a low incident intensity. Case C corresponds to the neck of the bowtie, with narrowest focus and highest irradiance. Case A represents an average irradiance condition.

To study the impact of axial nonuniformity we contrast the output performance between an axially uniform XRL with a nonuniform XRL simulating the Gekko focusing parameters. In the axially uniform case, we simulate a 3-cm-long XRL 


\begin{tabular}{|c|c|c|}
\hline Case & FWHM of focus $(\mu \mathrm{m})$ & Intensity $\left(\mathrm{TW} / \mathrm{cm}^{2}\right)$ \\
\hline A & 100 & 17 \\
\hline B & 150 & 11 \\
\hline C & 60 & 28 \\
\hline
\end{tabular}

Table .1. Irradiance conditions used for simulating the bowtie-shaped line focus geometry illustrated in Fig. 1. The cases correspond to the positions shown in Fig. 1.

with a straight line focus and an irradiance configuration defined by case $B$. The axially nonuniform run simulates the geometry shown in Fig. 1. For this study we chose to simulate only a snap shot corresponding to the peak of the 1-ns gaussian pulse and thus we are finding a "steady-state" solution to the evolution equation of matter and radiation.

Figures 3 contrast the 2-D gain profiles of cases $B$ and $C$ for the $G e J=2-1$ line at $236 \AA$. For case $C$, the narrow line focus results in larger $2-D$ plasma expansion with high temperatures and low densities. Since the gain is sensitively dependent on the local densities and temperatures, the wider line focus and lower intensity of case B actually results in a more ideal environment to achieve a spatially larger extent and more uniform Ge gain region. The density gradient, which impacts the refractive propagation of the XRL photons, is also more severe for case $\mathrm{C}$.

XRASER tracks the ray bundles through a dispersive media, in this case the XRL plasma, and keeps track of the intensity and directionality of each ray as it exits the plasma. The XRL footprint quickly evolves from a near-field, at the output face of the XRL, to a far-field pattern. The near-field footprint is dominated by the intensity at the output face and has a shape resembling of the gain distribution plotted in Fig. 3. The far-field footprint is determined by the angular distribution of the rays and is highly dependent on the density gradient.

To examine the XRL output, we take a spatial point in a far-field plane transverse to the axial direction and construct there an integral of XRL intensity over the differential solid angle. This integral quantity is shown in Fig. 4 as contour plots, comparing the spatially (a) uniform and (b) nonuniform far-field
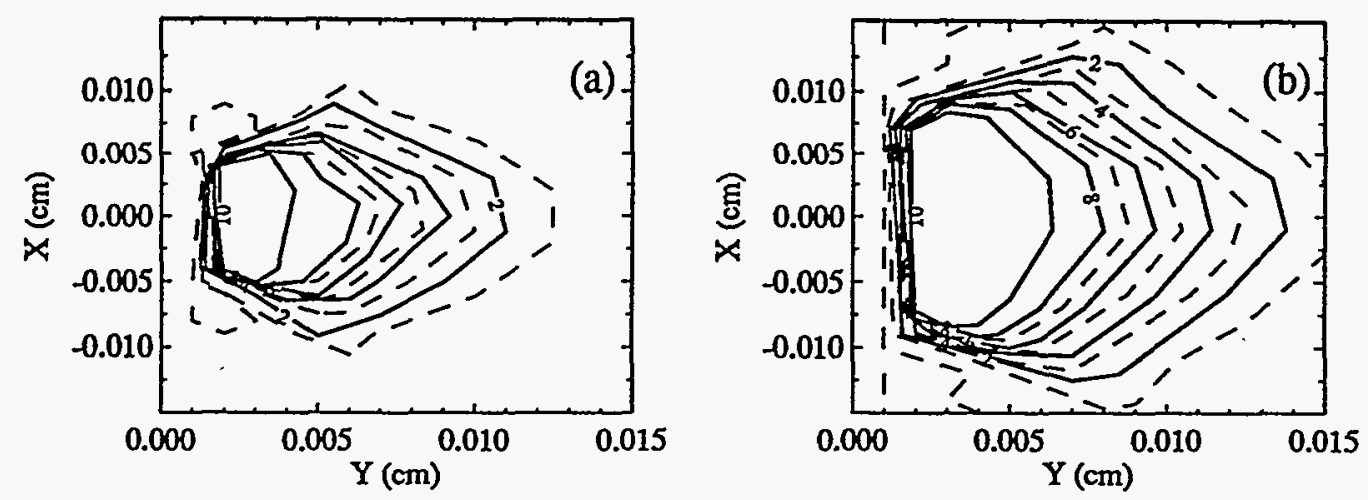

Figure 3. 2-D gain profiles of the Ge $236 \AA$ line at the peak of the driving laser pulse, for (a) narrow line focus (case $C$ ), and (b) wide line focus (case $B$ ). The solid lines are contours of gains at $2,4,6,8$, and $10 \mathrm{~cm}^{-1}$. 
footprints for the $236 \AA$ line, for all spatial points in the far-field plane. The coarseness of the angular bining for the XRL lines is evident in the figures. But we can still obtain qualitative comparisons between the two cases and discuss the impact of spatial nonuniformity on XRL output. Keep in mind that nonuniformity does not directly translate to worst XRL performance. There are instances where we want to vary the drive pulse along the XRL axis. One example is in an optical architecture [8] with an oscillator driving an amplifier where, by using different components axially, we can use the small aperture oscillator to drive the amplifier to gain higher laser output with improved coherence. Another example is the concept of adaptive spatial filtering of an XRL using geometric shaping to control the laser aperture and improve the transverse coherence of an XRL. [9]

The peak intensity of the spatially uniform case is a factor of 3 stronger than the nonuniform case for the $236 \AA$ line and $\sim 7$ for the $196 \AA$ line. Even with crude angular bining, we can still get some qualitative information from the far-field footprints. The beam divergence of the $236 \AA$ line, for both spatially uniform and nonuniform cases, are $\sim 6 \mathrm{mrad}$ FWHM in the blowoff direction (y-direction). We also calculated similar divergence for the $196 \AA$ line. This value is in reasonable agreement with the experimentally measured profiles. [2] The XRL divergence in the transverse (or $\mathrm{x}$-direction) for the uniform case is slightly wider despite lower density gradients, $\sim 10-12 \mathrm{mrad}$ for the 196 and $236 \AA$ lines. This is due to the large gain region in the $\mathrm{x}$-direction as shown in Fig. 3(b). Similar wide transverse footprints had been measured on Ge XRL experiments at Lawrence Livermore National Laboratory (LLNL). $[9,10]$ For the spatially nonuniform case, the transverse footprint is narrower, of order 6-8 mrad for the two XRL lines. One puzzle is the location of the centers of the laser footprints about the z-axis despite refraction. We expect the XRL to steer $\sim 8-10 \mathrm{mrad}$ off-axis due the large $n_{e}$ gradient. This result may be due to the coarse angular bining of the photons. We plan to performance further study on that and report it in later publications.

In a 3-D calculation, XRL photons will refractively propagate through regions of varying gain. The refraction angle is dependent on the local index of refraction, which is close to 1 . Since the XRL intensity is growing exponentially as a function of the gain-length product, the output is dominated by the rays that attain the largest gain lengths. For the spatially nonuniform case, the size of the gain media and the
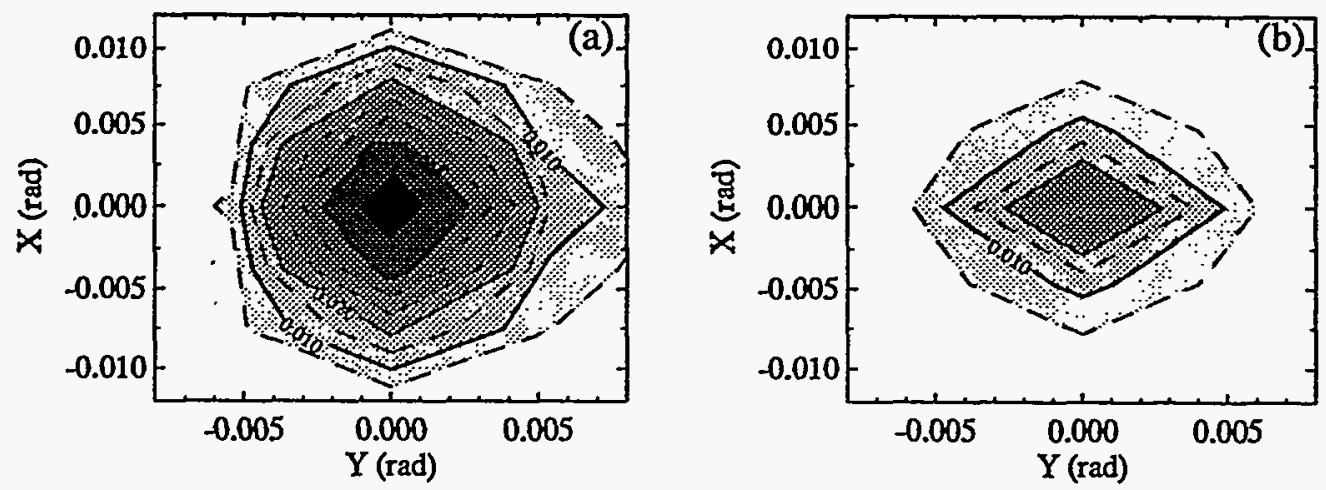

Figure 4. Far-field footprints of the spatially (a) uniform and (b) nonuniform cases for the $236 \AA$ line. The scale of the contour fill indicates the intensity level. 
density gradient are varying as XRL photons propagate along the axial direction. As shown in Fig. 3, the necks of the bowtie, represented by case $C$, has a smaller gain region. Without refraction; we can envision the necks of the bowtie acting as a spatial filter. The rays that traverse across the high gain region in the neck region will attain higher gain length and dominate the output of the spatially nonuniform case. However, with refraction, rays can refract in and out of the gain media and we must rely on 3-D calculations to quantify the impact of 3-D nonuniformity. And as evident by the comparison between the two 3-D simulations, we still observe significant output degradation with a bowtie-shaped line focus configuration.

\section{SUMMARY}

In this paper we studied the effect of axial nonuniformity on the output of XRLs, using the line focusing geometry of Gekko XII as an example. We contrast the output performance between an axially uniform XRL with a nonuniform XRL and observe significant degradation of the XRL output for the spatially nonuniform case. The output reduction is most likely due to axially varying spatial gain profile due to the difference irradiance configuration. In a 3-D dispersive gain media, rays that attain the largest gain length will dominate the output. The neck region of the bowtie-shaped line focus has a smaller gain region with larger density gradient. Rays that refracted out to lower gain region in the necks of the line focus will attain lower gain length product.

The complexity of a 3-D simulation is limited by the available memory and cpu. To simulate a "realistic" geometry, we were forced to use a simplified atomic model, coarse 3-D spatial mesh, and coarse photon angular bining. All these simplifications result in numerical uncertainties. We consider this calculation as a pioneering effort, testing the present day capabilities of computers and modeling codes in simulating a full 3-D laser problem. We plan to conduct detailed error analysis of 3-D simulations in the future as well as re-examine the impact of 3-D nonuniformity on the output of XRLs.

\section{ACKNOWLEDGMENTS}

Work performed under the auspices of the US DOE by LLNL under the contract \# W-7405-ENG-48.

\section{REFERENCES}

1. J. Nilsen et al., Phys. Rev: A 48, $4682-4685$ (1993).

2. R. Kodama et al., Phys. Rev. Lett. 73, 3215-3218 (1994).

3. Y. Kato et al., Proc. Ultrashort Wavelength II, SPIE 2012, 12 (1993).

4. G. B. Zimmerman et al., Com. Plasma Phys. Cont. Fusion 2, 51 (1975).

5. H. E. Dalhed, H. A. Scott, R. W. Mayle, private communications.

6. P. L. Hagelstein, LLNL-Report UCRL-53100 (January 1981).

7. R. W. Mayle, LLNL Report Nó. UCRL-JC-105280 (November 1990).

8. A. S. Wan et al., Optical Engineering 33, 2434 (1994).

9. A. S. Wan et al., Proc. of the 4th International Colloquium on X-Ray Lasers, D. Matthews, D. C. Eder, ed, (Williamsburg, VA 1994)

10. G. M. Shimkaveg et al., Proc. Lasers '92; C. Wang, ed, (1993). 